\title{
Mindestsicherungselemente in der gesetzlichen Rentenversicherung?
}

Die Ausrichtung der Diskussionen um die Weiterentwicklung der Gesetzlichen Rentenversicherung (GRV) hat sich in den vergangenen fünf Jahren gravierend verschoben. Waren die Rentenreformen von 2001 und 2004 noch von der Zielsetzung geprägt, die Beitragsbelastung der künftigen Generation soweit wie möglich zu begrenzen und dafür gegebenenfalls erhebliche Einschnitte bei den Leistungen der Rentenversicherung in Kauf zu nehmen, ist die aktuelle Diskussion vor allem auf die Frage gerichtet, ob das Leistungsniveau der GRV noch ausreicht, um einen deutlichen Anstieg der Altersarmut zu vermeiden. In Politik und Wissenschaft wird über Maßnahmen nachgedacht, mit denen einer befürchteten Ausweitung der Armut im Alter begegnet werden kann.

\section{Einleitung: Risiken steigender Altersarmut}

Armut im Alter stellt aktuell ein noch vergleichsweise geringes gesellschaftliches Problem dar. Der Anteil der Menschen, die staatliche Fürsorgeleistungen in Anspruch nehmen müssen, weil ihr Einkommen geringer ist als das gesetzlich definierte Existenzminimum, ist bei den älteren Menschen deutlich geringer als im Bevölkerungsdurchschnitt. Ende 2006 bezogen knapp $3 \%$ der über 65-Jährigen Leistungen der verschiedenen bedürftigkeitsgeprüften Grundsicherungssysteme (Grundsicherung im Alter und bei Erwerbsminderung, Grundsicherung für Arbeitsuchende, Hilfe zum Lebensunterhalt). In der Gruppe der 15- bis 65-Jährigen lag der Anteil der Grundsicherungsempfänger dagegen bei mehr als $10 \%$, bei den unter 15-Jährigen sogar bei fast $17 \%$. Es geht in der aktuellen Diskussion also nicht darum, Maßnahmen zur Behebung eines aktuell virulenten Problems einzuleiten, sondern um sinnvolle Ansätze, einen zukünftigen Anstieg der Altersarmut zu vermeiden. Deshalb bleibt die Zeit und somit auch die Chance, entsprechende Gegenmaßnahmen sorgfältig vorzubereiten, indem sie auf Basis einer fundierten Problemanalyse entwickelt werden.

Eine solche Problemanalyse könnte u. a. Erkenntnisse nutzen, die bei der Inanspruchnahme der „Grundsicherung im Alter und bei Erwerbsminderung" erworben wurden. Dieses Leistungssystem ist 2003 quasi als „Ersatz“ für die Sozialhilfe eingeführt worden, um den Eintritt von Altersarmut im Einzelfall durch subsidiäre, bedürftigkeitsgeprüfte Transferleistungen zu verhindern. Die aktuellen Zahlen zur Inanspruchnahme dieser Leistungen zeigen, dass das Armutsrisiko im Falle einer vorzeitigen Erwerbsminderung heute deutlich höher ist als das der Altersarmut. ${ }^{1}$ Die Notwendigkeit des Grundsicherungsbezuges im Falle einer vorzeitigen Erwerbsminderung führt aber in aller Regel dazu, dass auch im Alter ein Grundsicherungsbedarf fortbesteht. In der Zukunft könnte hieraus ein erhebliches Altersarmutsrisiko resultieren.

Auffällig ist ferner, dass Personengruppen, die nicht obligatorisch in die Gesetzliche Rentenversicherung (GRV) oder andere Alterssicherungssysteme eingebunden sind, überproportional häufig Grundsicherungsleistungen erhalten. So beziehen nur etwa zwei Drittel der Grundsicherungsempfänger im Alter von 65 oder mehr Jahren eine Altersrente aus der GRV. Nur für diesen Personenkreis trifft also das Bild einer „zu niedrigen“ gesetzlichen Rente, welche durch Grundsicherungsleistungen aufgestockt werden muss, zu. Etwa ein Drittel aller Bezieher einer Grundsicherung im Alter verfügt dagegen über keinerlei Leistungen aus der GRV (Rische 2008a, S. 3f.), während dies in der Gesamtbevölkerung in den alten Ländern für weniger als $10 \%$, in den neuen Ländern sogar nur für etwa $1 \%$ der Menschen der Fall ist (ASID 2007, S. 29ff.)

Im Hinblick auf die Ursachen eines in Zukunft möglicherweise steigenden Armutsrisikos liefert auch die Studie „Altersvorsorge in Deutschland 2005" (AVID 2005) $)^{2}$ interessante Hinweise. Danach weisen die Versicherungsbiografien der Menschen, die im Alter voraussichtlich mit einem besonders geringen Einkommen rechnen müssen, im Schnitt zwei- bis dreimal so lange Zeiten einer selbstständigen Tätig- keit und Zeiten der Arbeitslosigkeit auf wie Versicherte mit höheren Alterseinkommen (AVID 2005, S. 247ff.). Erwerbsbiografien, die durch lange Zeiten der Selbstständigkeit oder der Arbeitslosigkeit gekennzeichnet sind, implizieren offensichtlich ein deutlich erhöhtes Risiko, im Alter ein stark unterdurchschnittliches Einkommen zu beziehen.

Dieser Beitrag diskutiert im Folgenden, welcher der aktuellen Vorschläge geeignet erscheint, das zukünftige Risiko einer steigenden Altersarmut einzudämmen. $\mathrm{Zu}-$ nächst werden Risikofaktoren für zukünftige Altersarmut aufgezeigt (Abschnitt 2), bevor Konzepte der Mindestsicherung vorgestellt (Abschnitt 3) und dann bewertet werden (Abschnitt 4). Ein Fazit (Abschnitt 5) rundet den Beitrag ab.

\footnotetext{
Von den rd. 682.000 Empfängern dieser Leistung bezogen Ende 2006 gut 311.000 - also fast $46 \%$ - Grundsicherung wegen Erwerbsminderung (Statistisches Bundesamt 2008). In der GRV sind dagegen weniger als $10 \%$ aller Versichertenrenten Erwerbsminderungsrenten.

2 Einen Überblick über Methode und wichtige Ergebnisse der AVID 2005 liefern Frommert et al. (2008).
}

Reinhold Thiede, Dr., Dipl.-Volkswirt, Leiter des Referates für Entwicklungsfragen der Sozialen Sicherheit sowie des Bereichs "Entwicklungsfragen der Sozialen Sicherheit und Altersvorsorge" in der Deutschen Rentenversicherung Bund. Arbeitsschwerpunkte: Weiterentwicklung der gesetzlichen Rentenversicherung und des sozialen Sicherungssystems; Verteilungseffekte der sozialen Sicherungssysteme; betriebliche und private Altersvorsorge. e-mail: reinhold.thiede@drv-bund.de 


\section{Ursachenadäquate Strategie zur Vermeidung von Altersarmut}

Vor dem Hintergrund der Tatsache, dass empirisch basierte Informationen über die Ursachen des Entstehens von Altersarmut bereits vorliegen, hat die Deutsche Rentenversicherung Bund im vergangenen Jahr das Konzept einer „Ursachenadäquaten Strategie zur Vermeidung von Altersarmut " zur Diskussion gestellt (Rische 2008b; Loose 2008; Loose/Thiede 2006; Thiede 2005). Dieser Ansatz basiert auf der grundsätzlichen Zielsetzung, den Eintritt von Altersarmut soweit wie möglich zu vermeiden, anstatt ihn als unabwendbar hinzunehmen und die eingetretene Armut dann nachträglich durch Transferleistungen zu kompensieren. Dabei soll an den erkennbaren Ursachen von Altersarmut angesetzt und durch gezielte Maßnahmen darauf hingewirkt werden, dass es gerade bei potenziell gefährdeten Personen nicht zu Altersarmut kommt.

Konkret werden vier Ursachenkomplexe für ein erhöhtes Armutsrisiko im Alter identifiziert, an denen sich Vorschläge für Maßnahmen zur Vermeidung von Altersarmut orientieren:

\subsection{LÜCKEN IN DER VERSICHERUNGS- BIOGRAFIE ALS ARMUTSRISIKO}

Seit Jahren lässt sich eine Ausweitung der nicht sozialversicherungspflichtigen Formen der Erwerbsarbeit beobachten. Selbstständige Tätigkeiten, auch auf Basis von Honorar- oder Werkverträgen, sowie Minijobs nehmen einen wachsenden Raum in den Erwerbsbiografien der Versicherten ein (Mückenberger 1985; Klammer/Leiber 2006). Da diese Formen von Erwerbsarbeit in der Regel weder in der gesetzlichen Rentenversicherung noch in einem anderen Alterssicherungssystem obligatorisch gesichert sind, sind Personen, deren Erwerbsbiografien überdurchschnittlich stark von derartigen Formen von Erwerbsarbeit geprägt sind, in besonderem Maße im Alter von Armut bedroht (AVID 2005, S. 247ff.). Ein wichtiger Ansatz zur Vermeidung eines künftigen Anstiegs von Altersarmut kann darin liegen, möglichst alle Formen von Erwerbsarbeit in ein obligatorisches Alterssicherungssystem einzubeziehen. Die
Deutsche Rentenversicherung Bund schlägt daher eine Ausweitung der GRV auf jene Selbstständigen vor, die bislang nicht obligatorisch gesichert sind (Rische 2008a). ${ }^{3}$

\subsection{LANGZEITARBEITSLOSIGKEIT ALS ARMUTSRISIKO}

Der in der AVID 2005 aufgezeigte Zusammenhang zwischen niedrigen Alterseinkünften und überdurchschnittlich langen Arbeitslosigkeitszeiten in der Erwerbsbiografie ist, neben anderen Gründen, vermutlich auch auf die rentenrechtliche Bewertung derartiger Zeiten zurückzuführen, die im Zuge mehrerer Rechtsänderungen in den vergangenen zehn Jahren deutlich reduziert wurde. Wer über längere Zeit seines Lebens arbeitslos und auf die Leistungen des Arbeitslosengeldes II angewiesen ist, sieht sich daher inzwischen einer deutlich gestiegenen Gefahr von Altersarmut gegenüber. Hier wäre eine Modifikation der rentenrechtlichen Bewertung von Langzeitarbeitslosigkeit ein möglicher Weg zur Reduzierung dieses Altersarmutsrisikos.

\subsection{INVALIDITÄT ALS ARMUTSRISIKO}

Wie oben dargestellt, bezieht gegenwärtig annähernd die Hälfte aller Empfänger von Grundsicherung im Alter und bei Erwerbsminderung die bedürftigkeitsorientierte Sozialleistung vor Erreichen des Rentenalters, d. h. wegen vorzeitiger Invalidität. Eine bessere Absicherung der Menschen im Falle einer vorzeitigen Erwerbsminderung könnte das Armutsrisiko bei erwerbsgeminderten Menschen vor und nach Erreichen des Rentenalters verringern. Angesichts des in den jüngsten Rentenreformen vorgenommenen Paradigmenwechsels in der Alterssicherungspolitik - weg von der Zielsetzung einer lebensstandardsichernden gesetzlichen Rente hin zu dem Konzept der Lebensstandardsicherung aus mehreren Säulen, bei dem sich grundsätzlich Leistungen aus allen drei Säulen zu einer Lebensstandardsicherung ergänzen sollen - sollten dabei grundsätzlich auch die kapitalgedeckten Säulen nicht aus der Verantwortung entlassen werden. Aus Sicht der GRV sollten deshalb entsprechende Sicherungsprodukte in der zweiten und dritten Säule so (weiter-)entwickelt werden, dass sie für möglichst alle Versicherten zugänglich und von den Konditionen her auch akzeptabel sind.

\subsection{BESCHÄFTIGUNG IM NIEDRIG- LOHNBEREICH ALS ARMUTSRISIKO IM ALTER}

In einem weitgehend an Lohn- und Beitragsäquivalenz ausgerichteten Alterssicherungssystem führt eine dauerhafte oder langjährige Beschäftigung mit geringem sozialversicherungspflichtigen Entgelt zwangsläufig zu vergleichsweise geringen Alterssicherungsansprüchen. Damit wird die derzeit beobachtbare Ausweitung des Niedriglohnsektors in Deutschland (Bundesregierung 2008, S. 68ff.) zu einer möglichen Ursache für einen künftigen Anstieg der Altersarmut. In diesem Zusammenhang gilt es allerdings festzuhalten, dass die Lohnfindung in erster Linie eine Aufgabe der Tarifparteien ist; dabei sollten auch die Auswirkungen der Lohnfestsetzung auf die daraus abgeleiteten Ansprüche in den Sozialversicherungen berücksichtigt werden. $\mathrm{Ob}$ und in welchem Umfang flankierende Regelungen im Bereich der GRV zur Vermeidung einer späteren Altersarmut bei Niedriglohnbeziehern sinnvoll und möglich sind, wäre sorgfältig zu prüfen.

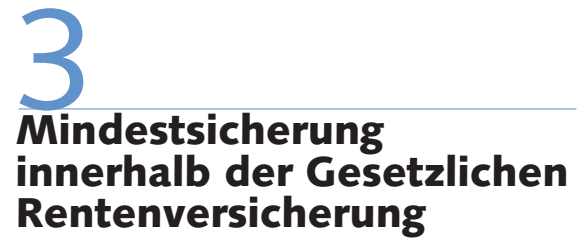

\subsection{ANSÄTZE}

Die Einbeziehung aller sonst nicht obligatorisch gesicherten Erwerbstätigen in die GRV oder eine bessere rentenrechtliche Bewertung von Zeiten der Langzeitarbeitslosigkeit könnten durchaus, da auf die Vermeidung von Altersarmut hin ausgerichtet, als Mindestsicherungselemente der Alterssicherung betrachtet werden. In der aktuellen Diskussion wird dieser Begriff aber zumeist sehr viel enger allein auf solche Maßnahmen bezogen, die unmittelbar an die Regelungen zur Leistungshöhe in den einzelnen Sicherungssystemen anknüpfen.

Grundsätzlich kann man dabei Ansätze zum Ausbau der Mindestsicherung in der Alterssicherung, die entsprechende Regelungen innerhalb der GRV vorsehen, von

\footnotetext{
3 Vor Kurzem wurde eine erste Abschätzung der Auswirkung eines solchen Ansatzes auf die Finanzsituation der Deutschen Rentenversicherung durchgeführt (Windhövel et al. 2009).
} 
solchen unterscheiden, die außerhalb der GRV angelegt sind. Beispielhaft für Mindestsicherungselemente innerhalb der GRV sind etwa folgende, derzeit diskutierte Ansätze:

- Die-gegebenenfalls modifizierte-Fortführung der nach geltendem Recht für Zeiten bis 1992 anzuwendende Regelung der "Mindestentgeltpunkte bei geringem Arbeitsentgelt“ (die sogenannte „Rente nach Mindesteinkommen"), die teilweise in den Gewerkschaften und der SPD präferiert wird.

- Eine allgemeine Aufstockung niedriger Renten auf einen Mindestrentenbetrag, die in jüngerer Zeit u. a. von Rürup (wieder) ins Gespräch gebracht wurde (Handelsblatt 2008).

- Eine stärkere Differenzierung der rentenrechtlichen Bewertung von Beitragszeiten in Abhängigkeit von der Höhe der bereits erreichten Rentenanwartschaften, also eine differenzierte Rentenformel, wie sie z. B. in Reformvorschlägen enthalten sind, die in der Partei DIE LINKE oder vom Deutschen Juristinnenbund diskutiert werden. Diese Ansätze gleichen teilweise den Regelungen des deutschen Rentenrechts vor der Rentenreform von 1957 oder der aktuellen Rentenformel in der Sozialversicherung der USA.

- Schließlich impliziert auch der Ansatz der „flexiblen Anwartschaften“, der in den späten 1990er Jahren im Bereich der damaligen Bundesversicherungsanstalt für Angestellte (BfA) entwickelt wurde (Langelüddeke et al. 1999; Thiede 2000), die Einfügung von Mindestsicherungselementen in die GRV.

Eine Mindestsicherung außerhalb der GRV stellt die bestehende bedürftigkeitsabhängige Grundsicherung im Alter und bei Erwerbsminderung dar. Bedürftigkeitsunabhängige staatliche Transfers als Form der Mindestsicherung sehen das seit den 1980er Jahren diskutierte und von Biedenkopf und Miegel entwickelte Konzept einer vorleistungsunabhängigen Grund- oder „Einheitsrente" (Miegel/Wahl 1999) oder den Ansatz des sogenannten Bürgergeldes („,bedingungsloses Grundeinkommen") vor. Letzteres ist eine vorleistungsunabhängige Transferzahlung für die gesamte Bevölkerung, also nicht nur für die Rentnergeneration (Borchard 2007).

Einen Ansatz zur Realisierung weitergehender Mindestsicherungselemente, der teilweise innerhalb und teilweise außerhalb der GRV angelegt ist, stellt schließlich das sogenannte Sockelrentenmodell der katholischen Verbände 4 dar. Dieses Modell enthält mit der außerhalb der GRV angesiedelten Sockelrente ein Element, das eine vorleistungsunabhängige Transferzahlung vorsieht, verbindet dies jedoch mit einer Pflichtversicherung innerhalb der GRV, die den heutigen Regelungen weitgehend entspricht.

Rentenversicherungsexterne Regelungen zur Vermeidung von Altersarmut sind grundsätzlich nicht an die dem Rentenrecht innewohnende Grundsystematik gebunden und können deshalb das Ziel der Armutsvermeidung möglicherweise zielgerichteter angehen als rentenversicherungsimmanente Regelungen. Auf der anderen Seite kann aber gerade dies zu Problemen führen, wenn die Regelungen der beiden Systeme nur eingeschränkt kompatibel sind. Als Beispiel hierfür können etwa die Fehlanreize gelten, die durch die Anrechnung von Renten der GRV (oder von Leistungen aus der betrieblichen Altersversorgung oder der privaten Vorsorge) auf die Leistungen der Grundsicherung im Alter und bei Erwerbsminderung entstehen: Bei Personen mit einem Gesamtalterseinkommen unterhalb des Grundsicherungsbetrages führen die gesetzliche Rente oder die Zusatzversorgung dann unter Umständen nur zu einer Minderung der Grundsicherungsleistung, nicht aber zu einem höheren Gesamteinkommen. Generell werden zudem bei vorleistungsunabhängigen Einheitsrentenmodellen negative Leistungsanreize erwartet.

Wegen der Themenstellung dieses Schwerpunktheftes, das den Fokus auf die Sozialversicherungen legt, beschränkt sich der vorliegende Beitrag im Folgenden darauf, Ansätze zu diskutieren, die eine Integration bzw. den Ausbau von Mindestsicherungselementen innerhalb der GRV beinhalten.

\subsection{RENTE NACH MINDESTEINKOMMEN}

Seit 1972 gibt es im deutschen Rentenrecht Regelungen, nach denen, bei Vorliegen bestimmter Voraussetzungen, die Rentenanwartschaften aus Zeiten mit niedrigem sozialversicherungspflichtigem Entgelt bei der Ermittlung der Rentenhöhe besser bewertet werden, als es dem zugrundeliegenden Arbeitsentgelt entspricht. Diese soge- nannte „Rente nach Mindesteinkommen“ sieht nach geltendem Recht vor, dass Rentenanwartschaften aus Pflichtbeiträgen vor 1992 um die Hälfte angehoben werden, sofern ein Versicherter 35 Jahre mit rentenrechtlichen Zeiten aufweist und der Durchschnittswert aller vollwertigen Pflichtbeiträge, also auch derjenigen für die Zeit ab 1992, niedriger ist als $75 \%$ des Wertes eines Durchschnittsverdieners. Die Anhebung der Rentenanwartschaften aus den entsprechenden Zeiten wird allerdings nach oben begrenzt: Maximal können durch die Regelung niedrige Rentenanwartschaften bis auf den Wert von $75 \%$ eines Durchschnittsverdieners angehoben werden. Die heute geltende Regelung der Renten nach Mindesteinkommen geht dabei auf die Rentenreform von 1992 zurück. Seinerzeit wurde die "Vorgängerregelung“, die nur Zeiten vor 1973 erfasste, in modifizierter Form auf Zeiten bis 1992 ausgeweitet.

\subsection{AUFSTOCKUNG NIEDRIGER RENTEN AUF EINEN MINDESTBETRAG}

Alternativ zu einer Verlängerung der „Rente nach Mindesteinkommen" werden Ansätze diskutiert, die vorschlagen, bei Vorliegen bestimmter Voraussetzungen niedrige GRV-Renten nicht um einen bestimmten Prozentsatz, sondern auf einen genau definierten Mindestbetrag (oberhalb der Grundsicherung) aufzustocken; allerdings soll die Aufstockung nach den bisher diskutierten Vorschlägen dabei auf langjährig Versicherte begrenzt sein. Solche Regelungen sollen gewährleisten, dass Versicherte aufgrund langjähriger Beitragszahlung zumindest ein höheres Alterseinkommen erlangen als Personen, die nie oder nur kurz Beiträge gezahlt haben und im Alter die Leistungen der Grundsicherung in Anspruch nehmen.

Mit diesem Ansatz will man u.a. auch negative Anreizwirkungen auf die Bereitschaft zur Beitragszahlung in der gesetzlichen Rentenversicherung und/oder zur zusätzlichen privaten Vorsorge vermeiden. Angesichts dieser Zielsetzung ist es folgerichtig, die Aufstockung der niedrigen GRV-Renten ohne Berücksichtigung der Bedürftigkeit des Betroffenen - und insbesondere auch ohne eine Anrechnung von anderen Alterseinkünften oder Vermögen -

4 "Solidarisch und gerecht: Das Rentenmodell der katholischen Verbände" (http://www.buendnissockelrente.de/Broschuere_Rentenmodell_12-2007). 
zu gestalten. Die Aufstockung wäre also kein bedingter Anspruch des Versicherten wie bei der Grundsicherung im Alter und bei Erwerbsminderung, sondern ein unbedingter Rechtsanspruch.

\subsection{DIFFERENZIERTE RENTENFORMEL}

Entsprechend dem Grundsatz der Lohnund Beitragsbezogenheit der Renten sind im deutschen Rentenrecht die von einem Versicherten in einem Jahr erworbenen Rentenanwartschaften grundsätzlich linear abhängig von seinem zugrundeliegenden sozialversicherungspflichtigen Entgelt im gleichen Jahr: Ein Versicherter, der (unterhalb der Beitragsbemessungsgrenze) doppelt soviel verdient wie ein anderer, erhält auch eine doppelt so hohe Rentenanwartschaft. Darüber hinaus ist die Höhe der Rentenanwartschaft, die aufgrund eines bestimmten versicherungspflichtigen Entgelts in einem Jahr erworben wird, unabhängig von der Höhe der im Verlauf des Erwerbslebens insgesamt erworbenen Rentenanwartschaften. Mindestsicherungselemente können grundsätzlich auch dadurch in die GRV eingefügt werden, dass die strikte Interpretation des Begriffs der Lohnund Beitragsäquivalenz relativiert und die Rentenformel insofern modifiziert wird.

Dies wäre etwa der Fall, wenn die Höhe der aufgrund eines bestimmten sozialversicherungspflichtigen Entgelts erworbenen Rentenanwartschaft davon abhängig gemacht wird, wie hoch die von dem betroffenen Versicherten im Erwerbsleben insgesamt erworbenen Anwartschaften sind: So könnte beispielsweise aufgrund eines gegebenen versicherungspflichtigen Entgelts eine höhere Rentenanwartschaft erworben werden, bis ein bestimmter Grenzwert an Anwartschaften überschritten ist; danach würde für das gleiche versicherungspflichtige Entgelt eine geringere Rentenanwartschaft erworben.

\section{5 "FLEXIBLE ANWARTSCHAFTEN“}

Das Ende der 1990er Jahre in der Bundesversicherungsanstalt für Angestellte (BfA) entwickelte Modell der „flexiblen Rentenanwartschaften " ähnelt in seiner Struktur und seiner Wirkungsweise den Ansätzen einer differenzierten Rentenformel. Vorgeschlagen wird, dass in den ersten Versicherungsjahren ${ }^{5}$ mit den regulären Rentenversicherungsbeiträgen über die normalen Rentenanwartschaften hinaus zusätzliche „flexible Anwartschaften“ erworben werden. Diese können dazu verwendet werden, Lücken in der Erwerbsbiografie zu schlieBen, Zeiten mit niedrigem Arbeitsentgelt aufzuwerten oder Rentenabschläge bei vorzeitigem Renteneintritt auszugleichen. Da „flexible Anwartschaften“ nur in den ersten Pflichtbeitragsjahren erworben werden, in den folgenden Beitragsjahren dagegen nicht mehr, ergibt sich auch in diesem Modell so etwas wie eine differenzierte Rentenformel: In den ersten Versicherungsjahren wird bei gleicher Lohn- und Beitragshöhe insgesamt eine größere Rentenanwartschaft erworben als in späteren Versicherungsjahren.

Die Zielsetzung dieses Modells ist nicht in erster Linie die Vermeidung von Altersarmut, sondern die Anpassung der GRV an den Wandel in der Arbeitswelt. Da aber gerade die Veränderungen der Arbeitswelt eine wesentliche Ursache für den möglichen künftigen Anstieg der Altersarmut darstellen, ist dieser Ansatz auch im Zusammenhang mit der Frage der Armutsvermeidung zu diskutieren.

\section{1 \\ Bewertung einer Mindest- sicherung in der GRV}

\subsection{KRITERIEN}

Will man die genannten Ansätze zur Integration mindestsichernder Elemente in die GRV bewerten, so ist von der Frage auszugehen, ob bzw. in welchem Umfang mit den jeweiligen Ansätzen das angestrebte Ziel, die Vermeidung von Altersarmut, erreicht wird und welcher Aufwand dazu erforderlich ist. Konkret: Es geht um die Effektivität und die Effizienz der verschiedenen Ansätze. Effektivität meint dabei das Ausmaß der Zielerreichung einer Maßnahme, während das Kriterium Effizienz darauf abstellt, diese Zielerreichung mit möglichst geringem Mitteleinsatz zu realisieren. ${ }^{6}$

Hinsichtlich des Bewertungskriteriums Effektivität ist zu untersuchen, in welchem Umfang Altersarmut durch die verschiedenen Ansätze tatsächlich vermieden werden kann. Dabei wird man generell davon ausgehen müssen, dass in das Regelwerk der GRV integrierte Regelungen zur Vermeidung von Altersarmut nur eingeschränkt effektiv sein können. Die deutsche Rentenversicherung ist eine individuelle, lohn- und beitragsbezogene Sozialversicherung; der Haushaltskontext der Versicherten und ihre Einkünfte neben dem sozialversicherungspflichtigen Entgelt (in der Erwerbsphase) bzw. der GRV-Rente (in der Altersphase) spielen grundsätzlich keine Rolle bei der Bemessung der Rentenhöhe. Für die Frage der Armutsvermeidung sind gerade diese Aspekte jedoch von erheblicher Bedeutung. Im Hinblick auf die Effektivität der hier diskutierten Ansätze kann deshalb allenfalls geprüft werden, ob die Rente aus der GRV eine Höhe erreicht, die für sich genommen bereits Armut vermeidet. Im Folgenden wird dies dann angenommen, wenn eine Rente oberhalb des durchschnittlichen Grundsicherungsanspruchs von ca. $625 €$ pro Monat ${ }^{7}$ liegt.

Hinsichtlich des Bewertungskriteriums Effizienz - also der Frage, welcher Mittelaufwand bei dem jeweiligen Ansatz zur Zielerreichung erforderlich ist - geht es zunächst um die unmittelbar erforderlichen Finanzmittel. Eine wichtige Rolle spielt die Frage, wie zielgenau ein Instrument ist, ob es gezielt nur diejenigen unterstützt, die ansonsten im Alter arm wären, oder auch andere Personen. Wichtig ist ferner, dass das Kriterium der Effizienz nicht statisch, sondern als Ergebnis eines dynamischen Prozesses verstanden wird. Wenn ein Ansatz zur Vermeidung von Altersarmut z. B. aufgrund seiner Anreizwirkungen Verhaltensänderungen auslöst, die in der Folge den für die Zielerreichung erforderlichen Aufwand verändern, so ist dies bei der Effizienzprüfung zu berücksichtigen. Darüber hinaus ist zu berücksichtigen, dass Effizienz im sozialpolitischen Sinne weiter als ein (enger) ökonomischer Effizienzbegriff gefasst wird, der sich vor allem auf die Minimierung monetärer Kosten konzentriert. Ein ,erweiterter“ Effizienzbegriff berücksichtigt dagegen u.a. auch die Auswirkungen, die die betrachteten Maßnahmen auf andere Politikbereiche und die Realisierung der dort angestrebten Zielsetzungen haben.

5 In den Umsetzungsbeispielen zu diesem Ansatz wurde meist davon ausgegangen, dass in den ersten 20 Beitragsjahren zusätzliche flexible Anwartschaften entstehen.

6 Zur Effektivität und Effizienz bei der Bewertung sozialpolitischer Maßnahmen z. B. Thiede (1990, S. 108 ff.); Schmidt (1988, S. 199)

7 Dies entspricht dem durchschnittlichen Anspruch der Empfänger von Grundsicherung im Alter und bei Erwerbsminderung einschließlich der Aufwendungen für die Wohnung (Statistisches Bundesamt 2008) 
Es liegt auf der Hand, dass die in diesem umfassenden Sinne verstandene Effizienz von Maßnahmen zur Armutsvermeidung nur mit erheblichem Aufwand abzuschätzen ist. Schon aus Platzgründen muss deshalb an dieser Stelle darauf verzichtet werden. Dieses Vorgehen erscheint aber auch von daher gerechtfertigt, als $\mathrm{m}$. E. aus sozialpolitischer Sicht das Kriterium der Effektivität vorrangig ist: Ein Ansatz zur Armutsvermeidung, der diesem Ziel nicht hinreichend nahekommt, kann auch bei geringem Kostenaufwand nicht als sozialpolitisch befriedigend angesehen werden. Vor diesem Hintergrund konzentriert sich die Betrachtung im Folgenden vor allem auf die Frage der Effektivität der hier beschriebenen Ansätze, d.h. darauf, ob und in welchem Umfang mit ihnen sichergestellt werden kann, dass Altersarmut vermieden wird.

\subsection{RENTE NACH MINDEST- EINKOMMEN}

Im Hinblick auf die geforderte Anwendung der Regelungen zur Rente nach Mindesteinkommen auch für Zeiten ab 1992 ist zu konstatieren, dass dadurch das Ziel der Vermeidung von Altersarmut zumindest für zwei Gruppen von Versicherten nicht realisiert werden dürfte. ${ }^{8}$ Explizit ausgeschlossen von der Regelung sind Versicherte, die das Kriterium der „langjährigen Versicherungszugehörigkeit" nicht erfüllen. Aus diesem Grunde ist die Regelung nicht geeignet, Altersarmut bei Personen zu verhindern, die über längere Phasen ihres Erwerbslebens eine nicht versicherungspflichtige Tätigkeit (selbstständige Erwerbsarbeit, Mini-Jobs, Praktika, aber auch Zeiten der Aus- oder Fortbildung bzw. des Rückzugs in die sogenannte „stille Reserve") ausüben. Gerade bei Personen mit derartigen „Patchwork-Biografien “ ist aber, siehe oben, von einem besonders großen Risiko der Altersarmut auszugehen; hier würde die Rente nach Mindesteinkommen allein keinen adäquaten Lösungsansatz bieten (zur Kombination von Reformelemente vgl. Abschnitt 5).

Gleiches gilt für Versicherte, die über längere Zeiten ihres Erwerbslebens sehr niedrige sozialversicherungspflichtige Entgelte bezogen haben. Da die Höherbewertung von Versicherungszeiten im Rahmen der Rente nach Mindesteinkommen proportional zur Höhe der in diesen Zeiten erzielten Arbeitsentgelte erfolgt, besteht die
Möglichkeit, dass Personen mit einer langen Verweildauer im Niedriglohnsektor trotz der Anwendung der Regeln der Rente nach Mindesteinkommen im Alter nicht auf eine Rente über dem Niveau der Grundsicherung kommen. Dass dies nicht nur eine theoretische Überlegung ist, belegt folgendes Beispiel: Nach Angaben des Deutschen Gewerkschaftsbundes (DGB) liegt das durchschnittliche Tarifgehalt von Friseuren in Sachsen bei 5,16€ pro Stunde oder $830 €$ im Monat. ${ }^{9}$ Wegen der generellen rentenrechtlichen Aufwertung der in den neuen Bundesländern erzielten Arbeitsentgelte ergibt sich daraus ein rentenrelevantes Jahreseinkommen von $11.820 €$; das entspricht einer Rentenanwartschaft von gut 0,38 Entgeltpunkten. Ein Versicherter, der 40 Jahre lang diese Entgeltposition eines Vollzeitfriseurs in Sachsen aufweist, kommt in den neuen Bundesländern (ohne die Regelungen der Rente nach Mindesteinkommen) auf eine Monatsrente von rund $370 €$ (Stand: 1.7.2009). Geht man fiktiv davon aus, dass die Regelungen der Rente nach Mindesteinkommen unbegrenzt in die Zukunft fortgeschrieben würden, käme man bei der gleichen Versicherungsbiografie auf einen monatlichen Rentenbetrag von $554 €$. Auch dieser Wert liegt deutlich unterhalb der Summe, die im Regelfall als Leistung der Grundsicherung beansprucht werden kann. Die Anwendung der Regelungen zur Rente nach Mindesteinkommen kann bei Versicherten mit sehr niedrigen Arbeitseinkommen also nicht verlässlich verhindern, dass zur Vermeidung von Altersarmut zusätzlich Grundsicherung beantragt werden muss.

\subsection{AUFSTOCKUNG NIEDRIGER RENTEN AUF EINEN MINDESTBETRAG}

Ansätze, bei denen niedrige Renten auf einen bestimmten Mindestbetrag aufgestockt werden, erscheinen zumindest in diesem Punkte gegenüber den Regelungen der Rente nach Mindesteinkommen effektiver: Auch Versicherte, die über längere Zeiten ihres Erwerbslebens sehr niedrige sozialversicherungspflichtige Entgelte bezogen haben, wären bei diesem Ansatz auf jeden Fall nicht mehr von Altersarmut betroffen. Allerdings wird auch bei diesen Regelungen das Ziel der Vermeidung von Altersarmut nur eingeschränkt erreicht, falls bestimmte Gruppen von Versicherten aus der Anwendung der Regelung ausgenommen werden. Das wäre etwa der Fall, wenn, wie bei den meisten in diesem Zusammenhang diskutierten Ansätzen, die Regelung nur für langjährig Versicherte oder für in Vollzeitarbeit tätige Versicherte angewendet würde. Dann würde wie bei der Rente nach Mindesteinkommen Altersarmut gerade bei jenen Personengruppen nicht verhindert, die wegen längerer Phasen der Ausübung einer selbstständigen Tätigkeit, eines Mini-Jobs oder anderer nicht sozialversicherungspflichtiger Tätigkeiten Lücken in der Versicherungsbiografie aufweisen. Insofern ist je nach konkreter Ausgestaltung auch bei Ansätzen, die eine Aufstockung niedriger Renten auf einen Mindestbetrag vorsehen, die Effektivität im Hinblick auf die Vermeidung von Altersarmut eingeschränkt.

\subsection{DIFFERENZIERTE RENTENFORMEL UND „FLEXIBLE ANWARTSCHAFTEN “}

Da der Ansatz der „flexiblen Anwartschaften" als Sonderform von Ansätzen einer differenzierten Rentenformel verstanden werden kann, sollen die beiden Ansätze hinsichtlich der Bewertung ihrer Effektivität gemeinsam betrachtet werden. Dabei ist zunächst gegenüber den beiden anderen Ansätzen positiv hervorzuheben, dass grundsätzlich alle Versicherten, unabhängig von der Anzahl ihrer Versicherungsjahre, von diesen Regelungen profitieren würden. Es würden also auch jene Gruppen von Versicherten, die aufgrund von „Patchwork-Biografien“ Lücken in ihren Versicherungsverläufen aufweisen, begünstigt. Allerdings kann weder der Ansatz der „flexiblen Anwartschaften“ noch das Modell der differenzierten Rentenformel in jedem Einzelfall garantieren, dass tatsächlich eine Rente oberhalb der Armutsgrenze erzielt wird. Auch hier kann bei Versicherten mit sehr geringen sozialversicherungspflichtigen Entgelten und/oder einer sehr geringen Zahl von Versicherungsjahren trotz Anwendung dieser Regelungen der Rentenbetrag unterhalb der Armutsgrenze bleiben. Hinsichtlich dieses Problems sind die beiden genannten Ansätze mit der Rente nach Mindesteinkommen vergleichbar.

8 Dies gilt, sofern man die derzeit geltenden Regelungen unverändert auch für Zeiten ab 1992 anwendet.

9 http://www.mindestlohn.de/argument/ hintergrund/niedrigloehne-in-deutschland/ niedrigloehne-in-sachsen/friseure-in-sachsen/ 


\section{Schlussfolgerungen}

Im Ergebnis zeigt sich, dass alle betrachteten Formen von Mindestsicherungselementen innerhalb der gesetzlichen Rentenversicherung im Hinblick auf das Ziel der Vermeidung von Altersarmut Defizite hinsichtlich ihrer Zielerreichung (Effektivität) aufweisen. Mit keinem der Ansätze, zumindest in den derzeit diskutierten Ausformulierungen, kann allein sichergestellt werden, dass für weitgehend alle Versicherten oder zumindest für die am stärksten gefährdeten Gruppen Altersarmut verlässlich ausgeschlossen wird. Nun ist die vollständige Vermeidung von Altersarmut durch Maßnahmen innerhalb der Rentenversicherung ohnehin nicht zu erreichen, schon allein deshalb, weil Altersarmut auch durch besondere Bedarfslagen entstehen kann (z. B. bei stationärer Pflege), die realistischerweise nicht durch Rentenleistungen auszugleichen sind. Einschränkungen bei der Zielerreichung diskreditieren die Modelle also nicht generell, solange nicht andere Maßnahmen im Hinblick auf die Vermeidung von Altersarmut deutlich Erfolg versprechender sind. Als offene Flanke der meisten Reformvorschläge erweist sich in diesem Zusammenhang aber das Erfordernis einer langjährigen Versicherungszugehörigkeit, das Versicherte mit sogenannten Patchwork-Biografien praktisch von der armutsvermeidenden Wirkung ausschließt.
Dieses Problem könnte allerdings erheblich entschärft werden, wenn der in der GRV gesicherte Personenkreis auf alle bislang nicht obligatorisch abgesicherten Erwerbstätigen ausgeweitet würde. Aktuelle, empirisch basierte Modellrechnungen zeigen, dass sich durch die Einbeziehung von bislang nicht gesicherten Selbstständigen in die GRV deren Alterseinkommen, je nach unterstellter Beitragszahlung der Betroffenen, unter Umständen deutlich erhöht. Der Anteil der Fälle mit hohem Altersarmutsrisiko kann so gegebenenfalls nennenswert reduziert werden (Frommert/ Loose 2009; Ehler/Frommert 2009). Darüber hinaus dürfte sich auch bei einer besseren Bewertung von Zeiten der Langzeitarbeitslosigkeit die Anzahl der Versicherten mit Rentenansprüchen unterhalb der Grundsicherungsschwelle erheblich vermindern.

Insofern scheint die Umsetzung des eingangs diskutierten Ansatzes der „ursachenadäquaten Strategie zur Vermeidung von Altersarmut" eine notwendige, möglicherweise sogar eine hinreichende Voraussetzung dafür, dass der befürchtete Anstieg der Altersarmut in der Zukunft vermieden werden kann. Die Umsetzung dieses Ansatzes erscheint daher vorrangig. Selbst eine zeitlich parallele Ausweitung der mindestsichernden Elemente erschiene kaum zieladäquat, da mit der Einbeziehung der bislang ungesicherten Erwerbstätigen und der besseren Bewertung von Arbeitslosigkeitszeiten Ursachen und Strukturen der verbleibenden Sicherungsdefizite deutlich von den derzeit feststellbaren Problempoten- zialen abweichen und unter Umständen andere Lösungsansätze sinnvoll würden.

Wenn sich auch nach einer Einbeziehung aller Erwerbstätigen in eine obligatorische Alterssicherung und einer ausreichenden rentenrechtlichen Berücksichtigung von Zeiten der Arbeitslosigkeit zeigen sollte, dass bestimmte Gruppen der Bevölkerung in nicht akzeptablem Umfang von einem systematischen Altersarmutsrisiko betroffen sind, müsste in einem zweiten Schritt erneut über den Ausbau mindestsichernder Elemente in der Alterssicherung nachgedacht werden. Dabei sollte man angesichts der eingeschränkten Effektivität von Ansätzen, die eine Mindestsicherung innerhalb der GRV vorsehen, gegebenenfalls auch Modellalternativen prüfen, die eine Mindestsicherung außerhalb der GRV beinhalten oder eine Kombination aus Regelungen innerhalb und außerhalb der Rentenversicherung darstellen. Es spricht vieles dafür, dass diese Ansätze hinsichtlich ihrer Effektivität mindestsichernden Maßnahmen innerhalb der GRV zumindest ebenbürtig sein dürften. Allerdings ist für die Bewertung aller Ansätze zur Vermeidung von Altersarmut neben der Effektivität, wie eingangs betont, auch die Effizienz der jeweiligen Regelungen maßgeblich, insbesondere die Frage der Anreizkompatibilität. Dabei dürften sich gerade bei Ansätzen, die eine Mindestsicherung außerhalb der Rentenversicherung vorsehen - insbesondere bei einheitlichen, vorleistungsunabhängigen Transfersystemen -, ganz erhebliche Probleme auftun. 


\section{LITERATUR}

ASID (2007): Bundesministerium für Arbeit und Soziales (Hrsg.): Alterssicherung in Deutschland 2007 (Untersuchung von Infratest Sozialforschung TNS im Auftrag des Bundesministeriums für Arbeit und Soziales), Forschungsbericht 391Z, Berlin

AVID (2005): Deutsche Rentenversicherung Bund/Bundesministerium für Arbeit und Soziales (Hrsg.): Altersvorsorge in Deutschland 2005 (Forschungsprojekt von Infratest Sozialforschung TNS), DRV-Schriften Band 75, München

Borchard, M. (Hrsg.) (2007): Das solidarische Bürgergeld - Analyse einer Reformidee, Stuttgart

Bundesregierung (2008): Lebenslagen in Deutschland. Dritter Armutsund Reichtumsbericht der Bundesregierung, Bundestagsdrucksache $16 / 9915$ v. 30.06

Ehler, J./Frommert, D. (2009): Für eine Pflichtversicherung bei Selbstständigkeit ohne obligatorische Alterssicherung, in: Deutsche Rentenversicherung 1, S. 36-57

Frommert, D./Loose, B. (2009): Integration ungesicherter Selbständigkeit in die GRV: Notwendige Weiterentwicklung der Alterssicherung in Bismarck-Tradition?, erscheint in: Sozialer Fortschritt

Frommert, D./Ohsmann, S./Rehfeld, U. (2008): Altersvorsorge in Deutschland 2005 (AVID 2005) - Die neue Studie im Überblick, in: Deutsche Rentenversicherung 1, S. 1-19

Handelsblatt (2008): Rürup fordert Kurswechsel in der Rentenpolitik, in: Handelsblatt vom 2.1.

Klammer, U./Leiber, S. (2006): Atypische Beschäftigung und soziale Sicherung, in: WSI-Mitteilungen 5, S. 287-292

Langelüddeke, A./ Rabe, B./Thiede, R. (1999): Flexible Anwartschaften und Anwartschaftszeiten; in: Die Angestelltenversicherung 1, S. 7-13

Loose, B. L. (2008): Die Suche nach armutsvermeidenden Ansätzen in der Alterssicherung: Mehr Antworten als Fragen - Mehr Lösungen als Probleme?, in: RVaktuell 3, S. 79-87
Loose, B. L./Thiede, R. (2006): Alterssicherung: Auch in Zukunft armutsfest? - Optionen der Armutsprävention in der Alterssicherung, in: RVaktuell 12, S. 479-488

Miegel, M./Wahl, S. (1999): Solidarische Grundsicherung - Private Vorsorge: Der Weg aus der Rentenkrise, München Mückenberger, U. (1985): Die Krise des Normalarbeitsverhältnisses, in: Zeitschrift für Sozialreform 7, S. 415-434 und Zeitschrift für Sozialreform 8, S. 457-475

Rische, H. (2008a): Weiterentwicklung der gesetzlichen Rentenversicherung zu einer Erwerbstätigenversicherung, in: RVaktuell 1, S. 2-10

Rische, H. (2008b): Bericht vor der Vertreterversammlung der Deutschen Rentenversicherung Bund am 25.6. in Münster, http://www.deutscherentenversicherung.de/ nn_4796/DRV/de/Inhalt/Presse/Reden/2008_ w_drv_bund_25_6__muenster/rede_rische.html

Schmidt, M. G. (1988): Sozialpolitik: Historische Entwicklung und internationaler Vergleich, Opladen

Statistisches Bundesamt (2008): Zahl der Grundsicherungsempfänger steigt weiter; Pressemitteilung vom 31.10.

Thiede, R. (1990): Die gestaffelte Pflegeversicherung - Sozialpolitische und ökonomische Aspekte eines neuen Modells, Frankfurt/New York Thiede, R. (2000): Flexible Anwartschaften: Modell zur Modernisierung des Rentensystems, in: Soziale Sicherheit 2, S. 48-52

Thiede, R. (2005): Alterssicherung muss sich lohnen - Ansätze für einen besseren "Sozialhilfe break-even " in der gesetzlichen Rentenversicherung, in: RVaktuell 12, S. 519-525

Windhövel, K./Funke, C./Möller, J.-C./Thiede, R./Ehler, J. (2009): Szenarien einer Eingliederung der Selbstständigen ohne obligatorische Altersvorsorge in die gesetzliche Rentenversicherung (Forschungsprojekt der Prognos AG in Zusammenarbeit mit der Deutschen Rentenversicherung Bund), erscheint in: DRV-Schriften 